\title{
Tear Resistance, FTIR and Morphology Study of Tree Pruning Waste Papers: Effect of Soda Pulping Concentration
}

\author{
Lee Zheng Xun ${ }^{1}$, Jiun Hor Low ${ }^{1}$, Lim Joon Hoong ${ }^{1}$, Rohah A. Majid ${ }^{2}$ \\ ${ }^{1}$ School of Computer Science and Engineering, Faculty of Innovation and Technology, Taylor's University, Lakeside \\ Campus, No.1, Jalan Taylor's, 47500 Subang Jaya, Selangor, MALAYSIA. \\ ${ }^{2}$ School of Chemical and Energy Engineering, Faculty of Engineering, Universiti Teknologi Malaysia, 81310 Johor
Bahru, Johor, MALAYSIA.
}

*Corresponding Author

DOI: https://doi.org/10.30880/ijie.2021.13.06.006

Received 12 May 2020; Accepted 24 February 2021; Available online 31 August 2021

\begin{abstract}
With the aim to explore the use of tree pruning waste as replacement material in papermaking, a study was conducted to investigate the effect of soda pulping concentration on the tear resistance, FTIR spectroscopy and surface morphology of the fabricated papers. By varying the concentration of sodium hydroxide solution from $5 \%$ to $25 \%$, tree pruning waste papers with different tear resistance, FTIR spectroscopy and surface morphology were fabricated. The tree pruning waste papers with the optimum tear resistance $(73049.68 \mathrm{mN})$ was produced when the pulping medium was prepared at $20 \%$ of sodium hydroxide solution. As confirmed by the FTIR spectroscopy and morphological study, the pulp fibres with improved interlocking surface morphology was produced at $20 \%$ of sodium hydroxide solution. Beyond that, the tear resistance of the papers decreases subsequent to the cellulose degradation and fibre fibrillation. The present study confirms the use of tree pruning waste as an alternative in papermaking. Nonetheless, the soda pulping concentration must be properly regulated in order to optimise the performance of the fabricated paper products.
\end{abstract}

Keywords: Tree pruning waste, paper, sodium hydroxide solution, concentration, tear resistance, Fourier-transform infrared spectroscopy, morphology.

\section{Introduction}

The importance of paper and paper products in our daily lives has been directly reflected by the high consumption of paper products such as corrugated cardboard, packaging, books and wallpaper. According to a statistical study [1], paper production reached a record high of 400 million tonnes in 2014. More than half of the paper supply is mainly used in the United States, China and Japan, three of which had a combined consumption of 269 million tonnes. Notwithstanding, paper demand is expected to increase further and is anticipated to achieve 521 million tonnes per year as the human population increases in the future. [2].

The pulp and paper industry is one of the manufacturing industries that has a significant impact on the environment. This phenomenon is attributed to the use of the main raw material, namely wood fibres, which has disrupted the balance of carbon dioxide concentration in the atmosphere due to the deforestation. In addition, the use of wood fibres for papermaking has also contributed to illegal harvesting, human rights, social conflicts and irresponsible plantation development [3]. Therefore, a great deal of efforts have been made to investigate the use of fibrous biomasses for papermaking in order to reduce dependence on wood fibres. These fibrous biomasses include rice straw [2], kapok fibre [4], napier grass [5], kenaf [6], pineapple leaves [7], oil palm empty fruit bunch [8], sago waste [9] and tea waste [4]. As reported by the scholars, these fibrous biomasses are not only successfully fabricated into papers, but also demonstrate their potential use in the development of other eco-friendly materials [10-21]. 
Nevertheless, the use of tree pruning waste for paper production is scarcely reported. Thus, it retains an unknown potential for papermaking. Also, unlike trees in the forests, tree pruning waste is free of charge as it is a type of garden waste. If tree pruning waste is proven can be used as the replacement material for papermaking, not only the cost of paper and pulp production can be reduced, but it also provides a solution to deforestation. With the aforementioned, the main aim of this work is to investigate the use of tree pruning waste as replacement material for papermaking. For this reason, the effect of concentration of sodium hydroxide solution (as the reaction medium of pulping process) on the quality of the fabricated papers was investigated and reported in the present study. The quality of the fabricated tree pruning waste papers was evaluated based on their tear resistance, Fourier-transform infrared (FTIR) spectroscopy and surface morphology.

\section{Materials and Methodology}

Tree pruning waste (type of trees: hardwood) was collected from the vicinity of Taylor's University as well as the housing area in Taman Mawar, Puchong, Selangor, Malaysia. The tree pruning waste was ground into small chips by using a grinder. Analytical grade sodium hydroxide (purity $>95 \%$ ) was supplied by Fisher Scientific. To prepare the pulping medium, sodium hydroxide pellets $(5 \%, 10 \%, 15 \%, 20 \%$ and $25 \%$ based on the mass of tree pruning waste) was dissolved in $4 \mathrm{~L}$ of distilled water and heated to $85 \pm 5^{\circ} \mathrm{C}$. The tree pruning waste $(100 \mathrm{~g})$ was then added into the sodium hydroxide solution. After 1 hour, the tree pruning waste pulp was filtered and dried in an oven at $60^{\circ} \mathrm{C}$. To produce the tree pruning waste papers, the dried pulp was softened by using tap water and spread over a sheet mold. After the sun drying process, hot pressing process $\left(75^{\circ} \mathrm{C}\right.$ and 5 tonnes) was applied to the fabricated tree pruning waste papers by using a hydraulic press to improve the contact and bonding among the pulp fibres. The tear resistance of the fabricated papers were evaluated by using an Elmendorf-type tearing tester (Brand and Model: Hung Ta, HT-8181) according to the procedures described in the ASTM D689. The measurement was repeated for ten times for each formulation and the average value was reported. The tearing force was calculated by using Equation (1).

Average tearing force, $\mathrm{mN}=(16 \times$ average scale reading $\times$ capacity, $\mathrm{N}) \div($ number of plies $\times 15.7 \mathrm{~N})$

The Fourier-transform infrared (FTIR) spectroscopy of the tree pruning waste raw fibres as well as the pulp fibres was studied by using an attenuated total reflectance-FTIR spectrometer (Perkin Elmer Spectrum 100). The spectra were recorded in a frequency range of $4000 \mathrm{~cm}^{-1}$ to $650 \mathrm{~cm}^{-1}$ at a resolution of $4 \mathrm{~cm}^{-1}$. The peak assignments were done based on the works reported by L. G. Wade [22], Low et al. [23] and Horikawa et al. [24]. A Hitachi S-3400N variablepressure scanning electron microscope was operated at an accelerating electron of $10 \mathrm{kV}$ to examine the surface morphology of the tree pruning waste raw fibres and papers (magnification: 500X and 100X). Prior the surface examination, the samples were coated with a thin layer of platinum by using an auto fine coater (Quorum Q150R S).

\section{Results and Discussion}

The tree pruning waste papers were successfully fabricated by using different concentration of sodium hydroxide solution as pulping medium. The tear resistance, FTIR spectroscopy and surface morphology of the tree pruning waste papers were then characterized and reported in section 3.1, 3.2 and 3.3, respectively.

\subsection{Tear Resistance}

The tear resistance of the tree pruning waste papers produced at different concentrations of sodium hydroxide solution is shown in Fig. 1. As demonstrated by the trend line, the tear resistance shows an increasing trend with the increase of concentration of sodium hydroxide solution up to $20 \%$. In other words, tree pruning waste paper with the highest tear resistance were produced when $20 \%$ sodium hydroxide solution was used for the pulping process. Beyond that, adverse effect is observed on the tear resistance of the papers. In fact, the elevation of the tear strength is attributed to the removal of impurities on the fibre surface, which increases the available surface area for inter-fibre bonding. Undoubtedly, this circumstance increases the ability of the papers to withstand higher tear strength without rupture. Moreover, the extent of delignification is also increased through the elevation of sodium hydroxide concentration, which in turns, improves the tear resistance of the fabricated papers. The lignin removal process is important in pulp and paper manufacturing since high lignin content makes the papers become brittle, and thus, lower the tear resistance. On the other hand, adverse effect is observed on the tear resistance when the papers were prepared with high concentration of sodium hydroxide solution. This phenomenon is ascribed to the degradation and dissolution of cellulose molecules under vigorous alkaline condition. Owing to the cellulose is the main component to contribute the supporting structure and strength to the fabricated papers, the degradation and dissolution of cellulose reduces the molecular weight of cellulose in the produced pulp, and thus, the tearing force of the tree pruning waste papers. Furthermore, the degradation and dissolution of cellulose molecules also disrupts the cellulose crystallinity, which in turn, facilitates the diffusion of sodium hydroxide into the tree pruning waste fibres to hydrolyse other cellulose molecules (especially the amorphous cellulose) into their monomer (glucose). This circumstance further reduces the tear strength of the pulp as well as the papers produced. As shown in Fig. 1, the tree pruning waste paper with the 
highest tear resistance $(73049.68 \mathrm{mN})$ was produced when the pulping medium was prepared at $20 \%$ sodium hydroxide solution. Beyond that, the tear resistance of the papers declines and become $52178.34 \mathrm{mN}$. On the other hand, it can be seen that right after the $10 \%$ sodium hydroxide solution, the tear resistance of the tree pruning waste paper slightly decreases to $46960.51 \mathrm{mN}$ at $15 \%$ sodium hydroxide solution. This observation is rationalised by the inconsistent thickness of the tree pruning waste paper produced by using the traditional handmade approach in this work. This argument is supported by the study conducted by Hanna Karlsson [25], whereby the thickness of the paper can have significant effect on the tear resistance.

\subsection{Fourier-transform Infrared (FTIR) Spectroscopy}

Fig. 2 shown the Fourier-transform infrared (FTIR) spectroscopy of the tree pruning waste raw fibres as well as the pulp fibres produced by using 5\%, 20\% and $25 \%$ of sodium hydroxide solution. Firstly, the residual lignin in the pulp fibres produced with $5 \%$ and $20 \%$ of sodium hydroxide solution is directly reflected by the intensity of the absorption bands. The characteristics peaks of the residual lignin were recorded at $3382.02 \mathrm{~cm}^{-1}, 2926.83 \mathrm{~cm}^{-1}, 2853.66 \mathrm{~cm}^{-1}$, $1724 \mathrm{~cm}^{-1}, 1634 \mathrm{~cm}^{-1}$ and $1036.59 \mathrm{~cm}^{-1}$, respectively. These absorption bands are attributed to the polymeric association of $-\mathrm{OH}$ group $\left(3382.02 \mathrm{~cm}^{-1}\right), \mathrm{C}-\mathrm{H}$ stretching $\left(2926.83 \mathrm{~cm}^{-1}, 2853.66 \mathrm{~cm}^{-1}\right), \mathrm{C}=\mathrm{O}$ stretching $\left(1724 \mathrm{~cm}^{-1}\right)$, $\mathrm{C}=\mathrm{C}$ stretching $\left(1634 \mathrm{~cm}^{-1}\right), \mathrm{O}-\mathrm{H}$ bending and $\mathrm{C}-\mathrm{O}$ stretching $\left(1036.59 \mathrm{~cm}^{-1}\right)$ of n-propyl side chains of lignin. As observed, the intensity of these absorption bands decreases when the concentration of sodium hydroxide solution was elevated from $5 \%$ to $20 \%$. This observation implies that the extent of delignification of the pulp fibres produced by using $20 \%$ sodium hydroxide solution is higher than the one produced with $5 \%$ sodium hydroxide solution. In fact, sodium hydroxide solution has been proven as the effective delignification agent for lignocellulosic biomass [26]. When the tree pruning waste raw fibres were subjected to the pulping process, the alkali-labile linkages between lignin and cellulose are cleaved by the sodium hydroxide and dissolve the lignin in the pulping medium. Moreover, the rate of lignin dissolution is also directly proportional to the concentration of sodium hydroxide solution. These phenomena justify the discrepancies between the spectra of the pulp fibres (5\% and 20\%), whilst they also support the tear resistance reported in section 3.1 .

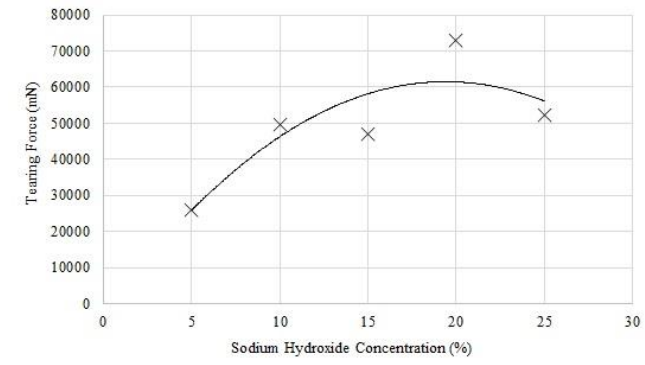

Fig. 1 - Tear resistance of papers fabricated at different concentration of sodium hydroxide solution

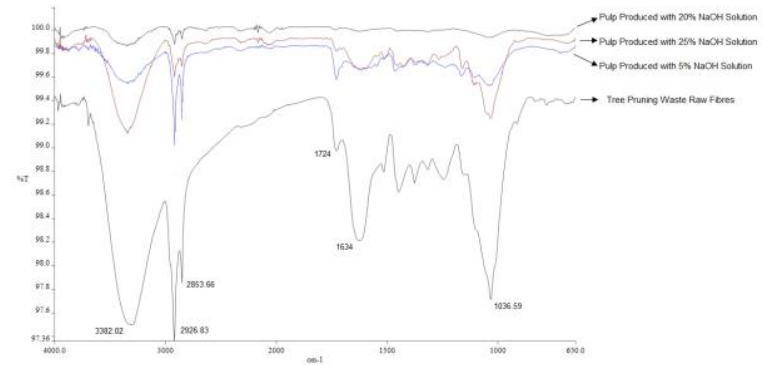

Fig. 2 - FTIR spectra of tree pruning waste raw fibres and pulp fibres

Nonetheless, adverse effect is observed on the intensity of the absorption bands $\left(3382.02 \mathrm{~cm}^{-1}, 2926.83 \mathrm{~cm}^{-1}, 2853.66\right.$ $\mathrm{cm}^{-1}, 1724 \mathrm{~cm}^{-1}, 1634 \mathrm{~cm}^{-1}$ and $1036.59 \mathrm{~cm}^{-1}$ ) when the concentration of sodium hydroxide solution was further elevated from $20 \%$ to $25 \%$. As explained, the rate of lignin dissolution is directly proportional to the concentration of sodium hydroxide solution. Thus, the intensity of these absorption bands should decrease due to the higher extent of delignification. When the tree pruning waste raw fibres were subjected to the high concentration of sodium hydroxide solution, not only lignin is removed from the fibres, but cellulose degradation also occurs during the pulping process and give rise to fibre fibrillation. The production of cellulose fibrils increases the specific surface area of the pulp fibre, which in turn, also increases the number of hydroxyl group ( $-\mathrm{OH}$ attached to the $\mathrm{C} 2, \mathrm{C} 3$ and $\mathrm{C} 6$ of cellulose) which can be found on the surface of the pulp. As a result, the intensity of the absorption band at $3382.02 \mathrm{~cm}^{-1}$ ( $-\mathrm{OH}$ group) increases when the pulp was produced by using $25 \%$ of sodium hydroxide solution. Owing to the $\mathrm{C}-\mathrm{OH}$ stretching (C6) of cellulose fibrils coincides with the absorption band of n-propyl side chains of lignin at $1036.59 \mathrm{~cm}^{-1}(\mathrm{O}-\mathrm{H}$ bending and $\mathrm{C}-\mathrm{O}$ stretching), this circumstance also gives rise to an increase in the intensity of this absorption band. On the other hand, the use of high concentration of sodium hydroxide solution during the pulping process also facilitates the oxidative alkaline degradation of the tree pruning waste fibres and result in the formation of carbonylcontaining oxidised celluloses. These carbonyl groups $(\mathrm{C}=\mathrm{O})$ are attributed to the formation of ketone groups, aldehyde groups and carboxyl groups in the celluloses during the oxidative alkaline degradation. The similar observation was also reported by Sjöström [27], Knill and Kennedy [28] in their alkaline degradation studies of biomass and cellulose. Thus, there is an increase of the intensity at $2926.83 \mathrm{~cm}^{-1}, 2853.66 \mathrm{~cm}^{-1}, 1724 \mathrm{~cm}^{-1}$ and $1634 \mathrm{~cm}^{-1}$ in the spectrum of the pulp (25\%) since these absorption bands are also the characteristic peaks of the $\mathrm{C}-\mathrm{H}$ stretching of aldehyde $\left(2926.83 \mathrm{~cm}^{-1}\right.$ and $\left.2853.66 \mathrm{~cm}^{-1}\right)$, carbonyl $(\mathrm{C}=\mathrm{O})$ stretching of ketone and carboxylic acid $\left(1724 \mathrm{~cm}^{-1}\right.$ and $\left.1634 \mathrm{~cm}^{-1}\right)$ of the oxidised celluloses. In other words, the FTIR spectroscopy of the pulp fibres is in line with the tear resistance, whereby the decrement of tear resistance at $25 \%$ sodium hydroxide solution is due to the degradation of cellulose in the pulp fibres. 


\subsection{Scanning Electron Microscopic Analysis}

The surface morphology of the tree pruning wastes as well as the pulp fibres produced was examined by using a scanning electron microscope (SEM). The micrographs of the tree pruning waste raw fibres as well as the pulp produced at $5 \%, 20 \%$ and $25 \%$ of sodium hydroxide solution are shown in Fig. 3, 4, 5, 6 and 7, respectively. As shown in Fig. 3 (a) and (b), the tree pruning waste raw fibres show a rough surface due to the adhesion of lignin [29] and other impurities such as wax, hemicellulose and dirt particles on the surface of the fibres. Moreover, there are also some splits and cracks accompanied by pieces of debris can be observed on the surface due to the fibre grinding process prior the pulp and paper manufacturing process.

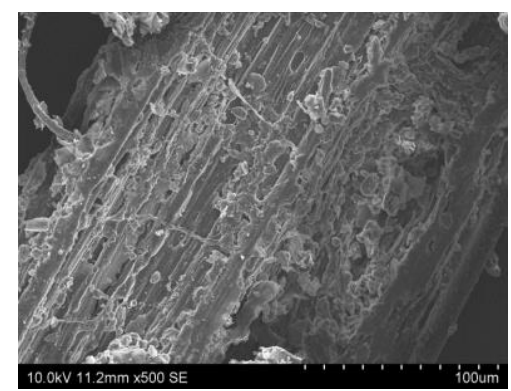

(a)

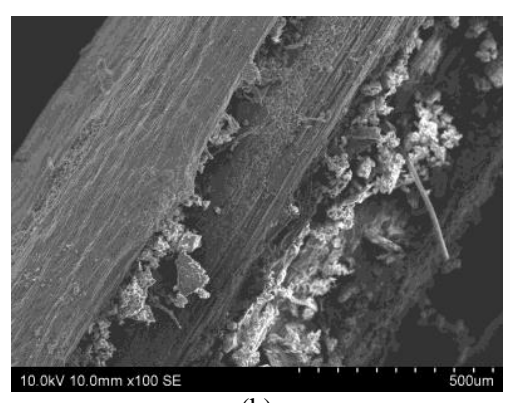

(b)

Fig. 3 - Scanning electron micrographs of tree pruning waste raw fibres at (a) 500X and (b) 100X magnification

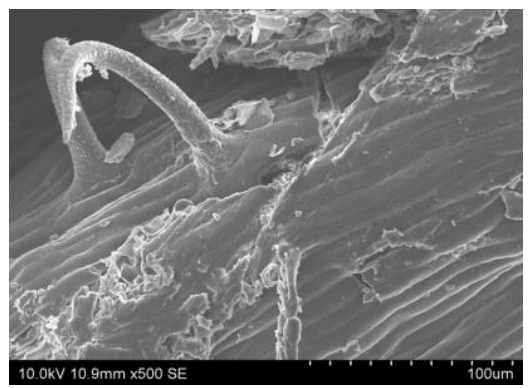

(a)

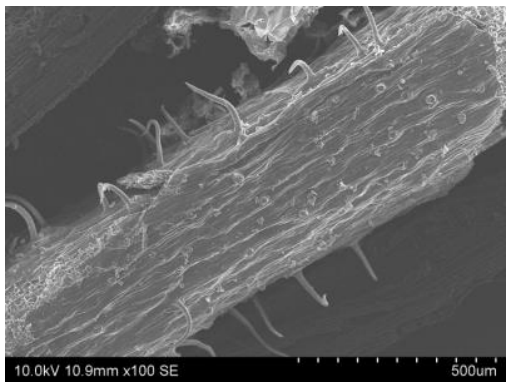

(b)

Fig. 4 - Scanning electron micrographs of pulp produced by using $5 \%$ sodium hydroxide solution at (a) 500X and (b) $100 \mathrm{X}$ magnification

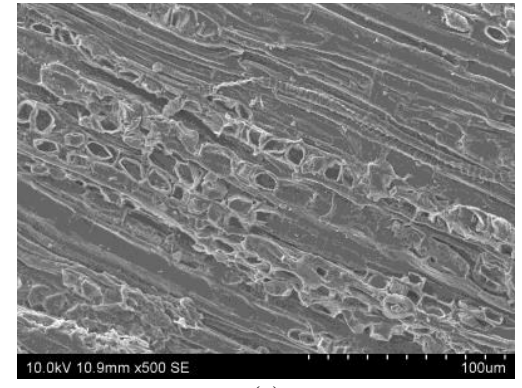

(a)

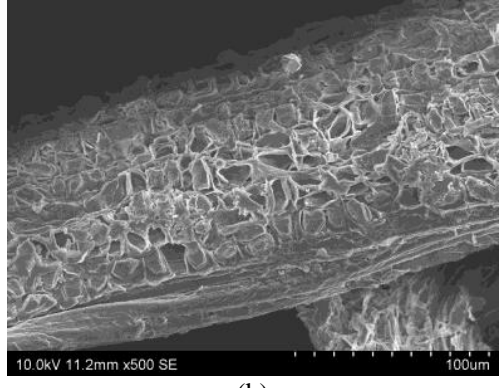

(b)

Fig. 5 - Scanning electron micrographs of pulp produced by using $20 \%$ sodium hydroxide solution at (a) $500 \mathrm{X}$ and (b) $100 \mathrm{X}$ magnification

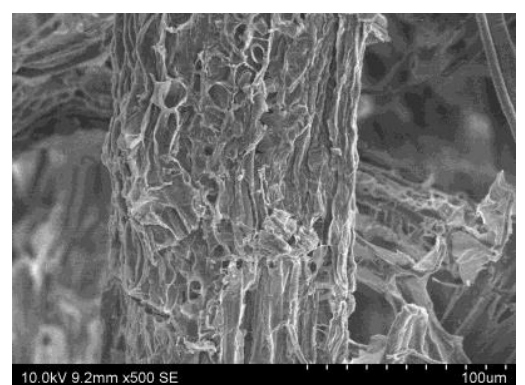

Fig. 6 - Scanning electron micrographs of pulp produced by using $25 \%$ sodium hydroxide solution

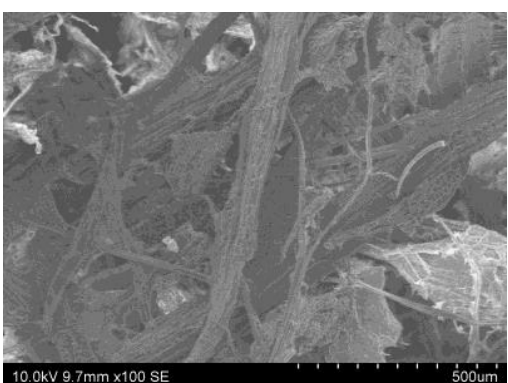

Fig. 7 - Fibrillation of pulp fibres produced by using $25 \%$ sodium hydroxide solution

By subjecting the tree pruning waste to the pulping process at 5\% sodium hydroxide solution, it can be observed that the surface of the pulp produced (Fig. 4 (a) and (b)) looks much cleaner and smoother compared to the raw fibres. In spite of there are some impurities still can be found on the surface of the pulp, the examination confirms that most of 
the lignin and impurities were removed during the pulping process by using sodium hydroxide solution. On the other hand, the surface cleanliness of the pulp was further improved when the concentration of sodium hydroxide solution was elevated to $20 \%$, as shown in Fig. 5 (a) and (b). This observation indicates the extent of delignification as well as the removal efficiency of impurities are improved with the increase of the concentration of sodium hydroxide solution. In addition, the surface of the pulp becomes rough accompanied by strong ridges compared to the one produced by using 5\% sodium hydroxide solution. This phenomenon is desired since the increased surface roughness and appearance of strong ridges provide better interlocking between fibres and promote the inter-fibre bonding, which in turns, provides beneficial effect on the mechanical strength of the fabricated papers. This argument is supported by Muhammad Arsyad [30], whereby the similar observation was reported when the coconut fibres were treated by using sodium hydroxide solution. Moreover, the surface examination of the pulp produced is also in line with the findings of tear resistance (section 3.1) and FTIR spectroscopy (section 3.2), since the tree pruning waste paper with optimum tear resistance is identified at $20 \%$ of sodium hydroxide solution.

In contrast, the surface of the pulp fibres produced by using $25 \%$ sodium hydroxide solution becomes rougher and followed by signs of flaking, as shown in Fig. 6. The surface changes is attributed to the degradation of cellulose due to the disruption of hydrogen bond network under vigorous alkaline condition during the pulping process. This argument is consolidated by the surface examination in Fig. 7. As recorded, the fibre bundle experienced fibrillation and degraded into fibrils when the concentration of the sodium hydroxide solution was increased to $25 \%$. This phenomenon reduces the strength which can be supported by the fibres without rupture, and thus, the strength of the fabricated papers. In other words, the examination of the surface morphology confirms the cellulose degradation as observed in the FTIR spectroscopy as well as the reduction in the tear resistance of the tree pruning waste paper.

\section{Conclusion}

Papers were successfully developed in this work by using tress pruning waste as the raw material. As evidenced by the tear test, FTIR spectroscopy and morphological study, the tear resistance of the fabricated tree pruning waste papers is directly influenced by the concentration of the sodium hydroxide solution. The tree pruning waste papers with the highest tear resistance were produced when $20 \%$ sodium hydroxide solution was used as the soda pulping medium. Under the optimum soda pulping condition, pulp fibres with better interlocking surface morphology were produced, and thus, the paper products. Adverse effect on the tear resistance is observed when the pulping concentration beyond $20 \%$. Under this pulping condition, cellulose degradation and fibre fibrillation is the main reason which depressed the tear resistance of the fabricated tree pruning waste papers. Tree pruning waste has demonstrated its potential for papermaking in the present work. To gain more insight into the effect of soda pulping concentration on the tree pruning waste papers, further study should focus on their physico-chemical properties.

\section{Acknowledgement}

This research was fully supported by Taylor's University [Project Code: TRGS/ERFS/1/2018/SOE/030] through Emerging Research Funding Scheme [Budget Code: 320201-SR67-429-C31].

\section{References}

[1] Haggith, M., Kinsella, S., Baffoni, S., Anderson, P., Ford, J., Leithe, R., Neyroumande, E., Murtha, N. \& Tinhout, B. (2018). The state of the global paper industry 2018. United States of America: Environmental Paper Network.

[2] Kaur, D., Bhardwaj, N. K. \& Lohchab, R. K. (2017). Prospects of rice straw as a raw material for paper making. Waste Management, 60, 127-139.

[3] World Wildlife Fund. 2019. Pulp and Paper. https://www.worldwildlife.org/industries/pulp-and-paper

[4] Majid, R. A., Rusman, R., Mohamad, Z., Zulkornain, A. A., Halim, N. A., Abdullah, M. \& Low, J. H. (2018). Development of tea waste/kapok fiber composite paper. Chemical Engineering Transactions, 63, 457-462.

[5] Lim, H. P., Rahman, W. A. W. A. \& Low, J. H. (2020). Properties characterization of napier grass (pennisetum purpureum) as the non-wood substitution for natural fiber papermaking. Journal of Advanced Mechanical Engineering Applications, 1, 27-35.

[6] Low, J. H., Ghanbari, T., Wan Abdul Rahman, W. A. \& A. Majid, R. (2018). Preparation and characterization of kenaf papers reinforced with tapioca starch: physicomechanical and morphological properties. Journal of Natural Fibers, 15, 191-203.

[7] Laftah, W. A. \& Abdul Rahaman, W. A. W. (2015). Chemical pulping of waste pineapple leaves fiber for kraft paper production. Journal of Materials Research and Technology, 4, 254-261.

[8] Faris, S. I., Ainun, Z. M. A. \& Jawaid, M. (2018). Effect of microcrystalline cellulose on the strength of oil palm empty fruit bunch paper. IOP Conference Series: Materials Science and Engineering, 368, 012042.

[9] Daud, W. S. A. b. W. M., Margaret Chan Kit, Y., Abdullah, N. \& Safrina Muhammad, A. (2010). Sago kraft paper: a potential solution to sago industry pollution. 2010 IEEE International Conference on Advanced Management Science (ICAMS 2010), 1, 80-83. 
[10] Sarah, S., Rahman, W. A. W. A., Majid, R. A., Yahya, W. J., Adrus, N., Hasannuddin, A. K. \& Low, J. H. (2018). Optimization of pineapple leaf fibre extraction methods and their biodegradabilities for soil cover application. Journal of Polymers and the Environment, 26, 319-329.

[11] Low, J. H., Andenan, N., Wan Abdul Rahman, W. A., Rusman, R. \& A.Majid, R. (2017). Evaluation of rice straw as natural filler for injection molded high density polyethylene bio-composite materials. Chemical Engineering Transactions, 56, 1081-1086.

[12] Low, J. H., Andenan, N. \& Wan Abdul Rahman, W. A. (2018). The influence of crosslink chemicals on the mechanical strength and water absorption of rice straw-based green composites. Journal of Natural Fibers, 15, 122-130.

[13] Ghazali, S. K., Lee, S. L., Low, J. H. \& Wan Abd Rahman, W. A. (2019). Properties of different composition of polyvinyl alcohol/sago waste biocomposite by extrusion processing. In S. H. Che Man \& N. Baharulrazi (Eds), Biomaterial based polymer composites: properties and characterization (pp. 11-26). Johor Bahru: Penerbit UTM Press.

[14] Low, J. H., Jusoh@Taib, E. R., Wan Abdul Rahman, W. A. \& S., W. (2019). Physicochemical properties of pineapple leaf fibres as textile material: effect of graft copolymerization of acrylonitrile. In S. H. Che Man \& N. Baharulrazi (Eds), Biomaterial based polymer composites: properties and characterization (pp. 97-112). Johor Bahru: Penerbit UTM Press.

[15] Pang, M. M., Aaron, Y., Koay, S. C., Low, J. H., Choo, H. L. \& Tshai, K. Y. (2019). Soil burial, hygrothermal and morphology of durian skin fiber filled polylactic acid biocomposites. Advances in Environmental Biology, $13,21-25$.

[16] Low, J. H. \& Wan Abdul Rahman, W. A. (2017). Plant fibers: renewable reinforcing filler in polyolefin biocomposites. In R. M. Kozlowski \&M. Muzyczek (Eds), Natural fibers: properties, mechanical behavior, functionalization and applications (pp. 303-324). New York: Nova Science Publishers, Inc

[17] Beh, J. H., Lai, J. C., Lim, T. H. \& Low, J. H. (2019). Synthesis and characterization of lithium iron phosphate nanowires from sago pith cellulose nanofibril aerogel. In R. Fangueiro (Eds), Proceedings of the 4th international conference on natural fibers - smart sustainable solutions (pp. 154-155). Porto: TecMinho - Associação Universidade-Empresa para o Desenvolvimento.

[18] Rusman, R., Majid, R. A., Rahman, W. A. W. A. \& Low, J. H. (2017). Carboxymethyl cassava starch/polyurethane dispersion blend as surface sizing agent. Chemical Engineering Transactions, 56, 1171-1176.

[19] Pang, M. M., Koay, S. C., Low, J. H., Buys, Y. F. \& Tshai, K. Y. (2018). Study on the plasticiser type for biobased microcrystalline cellulose filled polylactic acid composite. IOP Conference Series: Materials Science and Engineering, 458, 1-6.

[20] Rahman, W. A. W. A., Jusoh@Taib, E. R., Low, J. H. \& Mokeramin, M. (2015). Kopolimerisasi pencangkokkan akrilonitril pada serat daun nanas untuk kegunaan bahan tekstil. In Lembaga Perindustrian Nanas Malaysia (Ed), Seminar industri nanas 2015 "memperkasakan industri nanas negeri sarawak" (pp. 33). Kuching: Lembaga Perindustrian Nanas Malaysia.

[21] Wan Omar, W. N. A., Ghazali, S. K., Low, J. H. \& Wan Abd Rahman, W. A. (2019). Characterization of biodegradable corn starch/LDPE composite using maleic anhydride as compatibilizer. In S. H. Che Man \& N. Baharulrazi (Eds), Biomaterial based polymer composites: properties and characterization (pp. 43-56). Johor Bahru: Penerbit UTM Press.

[22] L. G. Wade, J. (2010). Organic Chemistry (7th ed.). USA: Pearson Prentice Hall.

[23] Low, J. H., Rahman, W. A. W. A. \& Jamaluddin, J. (2015). Structural elucidation of tannins of spent coffee grounds by CP-MAS 13C NMR and MALDI-TOF MS. Industrial Crops and Products, 69, 456-461.

[24] Horikawa, Y., Hirano, S., Mihashi, A., Kobayashi, Y., Zhai, S. \& Sugiyama, J. (2019). Prediction of lignin contents from infrared spectroscopy: chemical digestion and lignin/biomass ratios of cryptomeria japonica. Applied Biochemistry and Biotechnology, 188, 1066-1076.

[25] Karlsson, H. (2017). Some aspects on strength properties in paper composed of different pulps. Sweden: Karlstad University Studies.

[26] Kim, J. S., Lee, Y. Y. \& Kim, T. H. (2016). A review on alkaline pretreatment technology for bioconversion of lignocellulosic biomass. Bioresource Technology, 199, 42-48.

[27] Sjöström, E. (1991). Carbohydrate degradation products from alkaline treatment of biomass. Biomass and Bioenergy, 1, 61-64.

[28] Knill, C. J. \& Kennedy, J. F. (2003). Degradation of cellulose under alkaline conditions. Carbohydrate Polymers, 51, 281-300.

[29] Low, J. H., Xun, L. Z., Yoon, L. W., Pang, M. M. \& Wong, S. (2019). Exploration of tree pruning waste for papermaking. AIP Conference Proceedings, 2137, 020008.

[30] Arsyad, M. (2017). Effect of alkali treatment on the coconut fiber surface. ARPN Journal of Engineering and Applied Sciences, 12, 1870-1875. 